\title{
Measuring Family and Community Senses of Coherence in German
}

\author{
Senem Ezgi Vatandaslar², Ayse Rezan Cecen Erogul², Gernot Aich ${ }^{3}$ \\ ${ }^{I}$ This study is an excerpt from the first author's ongoing PhD thesis partially financed by the Scientific and \\ Technological Research Council of Turkey (TÜBITAK) and supervised by the second and third authors. \\ ${ }^{2}$ Mugla Sitki Kocman University \\ ${ }^{3}$ Pädagogische Hochschule Schwäbisch Gmünd
}

\begin{abstract}
Sense of coherence is one of the primary factors that help individuals deal with stressful situations. In the broadest sense, sense of coherence can be defined as the level at which an individual perceives the world to be comprehensible, manageable, and meaningful. Family sense of coherence and sense of community coherence are more specific concepts related to perceived comprehensibility, manageability, and meaningfulness of family and community, respectively. The study aims to adapt Family Sense of Coherence Scale and Sense of Community Coherence Scale to German and examine their psychometric properties in a sample of German university students. After obtaining necessary permissions from related authors and ethical board, the scales were translated from English to German by a fluent speaker of both languages. Then, four German scholars fluent in English and working in the field of psychology individually examined the translations and provided their opinions. Next, the authors corrected the items based on expert opinions. A pilot study was conducted where 10 students were asked to fill in the scales and express their opinions on clarity. The authors gave the scales their final form before applying them to 499 participants. The scales were examined in terms of internal consistency, composite reliability, construct validity, convergent and divergent validity, and measurement invariance. Findings are discussed in light of literature. As a result, it was concluded that German forms of Family Sense of Coherence and Sense of Community Coherence are valid and reliable in the sample of German university students.
\end{abstract}

Keywords: positive psychology; psychometrics; salutogenesis; scale adaptation; university students 